\title{
On the voltage and electric field distribution along polymer insulator
}

\begin{abstract}
In this paper, the outdoor polymer insulator was studied and the related electrical and mechanical conditions were reviewed. Moreover, different outdoor insulators were technically compared together. Furthermore, voltage distribution along a real polymer $33 \mathrm{kV}$ insulator was studied and the results were discussed accordingly. On the other hand, the voltage and electric field profiles at longitudinal and cross sections under different voltage levels were considered and discussed.
\end{abstract}

Keyword: Insulator; Polymer; Electric field 